\title{
Clinical guidelines from the Priority Epilepsy Program of the public health sector in Mexico
}

\author{
Juan C. Reséndiz-Aparicio
}

Hospital Psiquiátrico Infantil Dr. Juan N. Navarro y P.P.E, Instituto Nacional de Neurología y Neurocirugía Dr. Manuel Velasco Suárez. Mexico City, Mexico

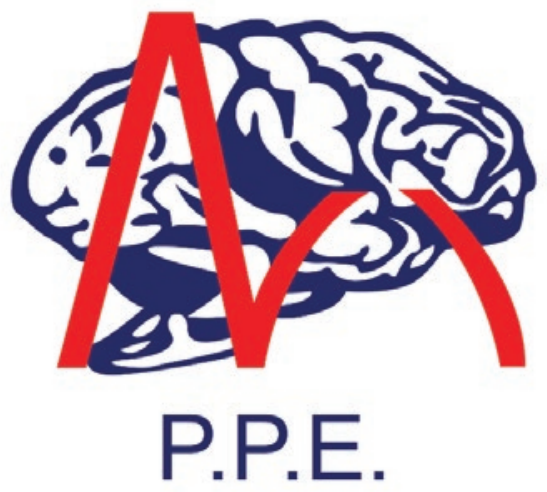

The Programa Prioritario de Epilepsia (PPE - Priority Epilepsy Program) was created based on the accord published in the Mexican Official Gazette of the Federation on October $24^{\text {th }}, 1984$. This program has labored in an uninterrupted manner to regulate, coordinate, methodize, and optimize the strategies in favor of patients with epilepsy, as well as their families and society. There are currently 78 centers of integral treatment for epilepsy in Mexico, located in various hospitals belonging to Mexico's health sector.

The headquarters for the national coordination is in the Instituto Nacional de Neurología y Neurocirugía (National Institute of Neurology and Neurosurgery) "Dr. Manuel Velasco Suarez" (INNN due to its acronym in Spanish) in Mexico City, from where all actions are planned for this task. The national coordination is led by its creator and founder Francisco Rubio Donnadieu, MD and by the author.

The development of the first Clinical Guidelines (CGs) has been a laborious effort, one that has been finished due to the work of all the coordinators of the PPE, who are neurologists and pediatric neurologists certified by the Mexican Board of Neurology and who work in one of the many institutions of the health sector in Mexico. To elaborate the CG, all the coordinators of the PPE met in person in two meetings, the first in the city of Leon and the second in the city of Puebla, where we formed workgroups for each CG. These meetings were possible due to the support of the federal government and the contributions of the

\section{Correspondence:}

Juan Carlos Reséndiz-Aparicio

E-mail: jc_doc@yahoo.com

1665-5044/@ 2019. Academia Mexicana de Neurología A.C. Published by Permanyer México. This is an open access article under the CC BY-NC-ND license (http://creativecommons.org/licenses/by-nc-nd/4.0/).

Date of reception: 01-02-2019

Date of acceptance: 28-02-2019 
pharmaceutical laboratories that aid in training primary health-care physicians. These CGs are designed to aid the primary health-care physicians and the specialists in making adequate decisions when approaching epileptic patients of different age groups and genders. These are the culmination of the experience of their authors, who have followed the necessary steps for proper and updated scientific research, using the criterion of the American Epilepsy Society 2016 to analyze the levels of evidence and recommendations including the benefit for the patients. To evaluate the quality of the CG, two experts coordinated each table and applied the Spanish version of the AGREE instrument of 2001.

Due to the advances in the knowledge of epilepsy, the PPE group aims to update the CG every 5 years.

These CGs constitute a series of recommendations developed by a group of medical physicians that have a particular interest in the field of epilepsy and work throughout the various institutions of the health sector; however, it is understood that the application of said recommendations depends on many factors. It is important to state that there are no conflicts of interest in these CGs due to the fact that they are editorially independent of any external funding.

Finally, I would like to thank the INNN for their hospitality and support in the coordination of the Program, the Mexican Academy of Neurology for the publication in their magazine, the Mexican Society of Pediatric Neurology who supported the process of translating the CG to English, and the authorities of the Hospital Psiquiátrico Infantil (Children's Psychiatric Hospital) of Mexico City who have allowed me to work as the adjunct executive member of the Priority Epilepsy Program and above all, thank you to the coordinators of the Priority Epilepsy Program who worked on this project; this work is dedicated to them and their families and to which I express my most ample recognition.

\section{Juan Carlos Reséndiz Aparicio, MD, Adjunct Executive Member}

\section{American Epilepsy Society 2016}

Article Classification: Evidence
Class I: A randomized, prospective and controlled clinical trial with masked outcome assessment, in a repre-
sentative population. The following are required:
a. No more than two specified primary results.
b. Blind allocation of subjects.
c. Exclusion/inclusion criteria are clearly defined.
d. Relevant baseline characteristics are presented and substantially equivalent among treatment
groups or there is appropriate statistical adjustment for differences.
e. Adequate accounting for dropouts with numbers sufficiently low to have a minimal potential for
bias. (study was completed with at least $80 \%$ of the enrolled subjects).
f. Demonstration of superior design of the studies or demonstration of non-inferiority with a $10 \%$
non- inferior design margin.
Class II: A randomized, prospective and controlled clinical trial with masked outcome assessment that lacks
one or two criteria of Class I a-e above, or a prospective matched cohort study with masked objec-
tive outcome assessment in a representative population that meets a-e.
Class III: All other control trials in a representative population, where outcome was independently assessed
by objective outcome measurement.
Class IV: Evidence from non-controlled trials, including series reports, case reports, consensus, or expert
opinion.




\begin{tabular}{|l|}
\hline \multicolumn{1}{|c|}{ Evidence for the } \\
Recommendation \\
Level A \\
One or more Class I trials or \\
two or more Class II trials. \\
Level B \\
One or more Class II trials or \\
three or more class III trials. \\
Level C \\
Two or more Class III trials. \\
Level U \\
Absence of trails that com- \\
plement levels A, B, or C. \\
Level R-PPE \\
\end{tabular}

\begin{tabular}{l} 
Conclusion and Recommendation \\
Conclusion, Level A \\
Established as effective, ineffective, or harmful for the given condition in \\
the specified population. \\
Recommendation: \\
Must be done or must not be done. \\
Conclusion, Level B \\
Probably effective, ineffective, or harmful for the given condition in the \\
specified population. \\
Recommendation: \\
It must be considered or must not be considered. \\
Conclusion, Level C \\
Possibly effective, ineffective, or harmful for the given condition in the \\
specified population. \\
Recommendation: \\
It could be considered or should not be considered. \\
Conclusion, Level U \\
Data is insufficient or inadequate given current knowledge, treatment is \\
unproven \\
Recommendation: Should not be performed \\
Conclusion, Level R-PPE \\
No evidence from levels A, B, or C, but it is a recommendation by con- \\
sensus of the group that elaborated the Clinical Guidelines of the PPE. \\
\hline
\end{tabular}

In all of the CGs of the Priority Epilepsy Program (PPE), the abbreviations we published are the same as the book "Epilepsia" by authors Rubio, Reséndiz, Alonso, and Sentíes, by the editorial Alfil in 2016, page numbers IX, X y XI; ISBN 978-607-741-168-0.

\section{Glossary and Abbreviations}

\section{Channelopathies}

SCN4A, SCN2A, SCN1B
KCNA1, KCNQ2, KCNQ3
CACNA1A
CHRNA4, CHRNB2
GLRA1
GABRG2

Ion: NA: sodium; K: potassium; $\mathrm{CA}$ : calcium; $\mathrm{CH}$ : acetylcholine; GL: glycine; GABA: gamma-aminobutyric acid

Channel or receptor: $\mathrm{CN}$ : channel; R: receptor; $\mathrm{N}$ : nicotinic

Subunit: A: $\alpha$; $B: \beta:$ : $;$ G: $\gamma$

\section{Seizure}

ES: epileptic seizure

GS: generalized seizure

GTCS: generalized tonic-clonic seizure

FS: focal seizure

FIAS: focal impaired awareness seizures (or disconnection from medium)

FAS: focal aware seizure/simple partial seizure FBTCS: focal to bilateral tonic-clonic seizure SE: status epilepticus

FeS: febrile seizure

\section{Electrolytes and neurotransmitters}
$\mathrm{Ca}^{++}$: calcium
$\mathrm{Cl}^{-}$: chlorine
$\mathrm{K}^{+}$: potassium
$\mathrm{Mg}^{++}:$magnesium
$\mathrm{Na}^{+}$: sodium

GABA: gamma-aminobutyric acid NMDA: N-methyl-D-aspartate

AMPA: a-amino-3-hydroxy-5-methyl-4-isoxazolepropionic acid 


\section{Neurologic structures}

BBB: blood-brain barrier

CSF: cerebrospinal fluid

CNS: central nervous system

\section{Diagnostic tests}

fMRI: functional magnetic resonance imaging

MRI: magnetic resonance imaging

PET: positron emission tomography

SPECT: single-photon emission computed tomography

CT: computed tomography scan

ECoG: electrocorticography/intracranial electroencephalography

EEG: electroencephalogram

MEG: magnetoelectroencephalogram

Video-EEG: video electroencephalogram

PSG: polysomnogram

EKG: electrocardiogram

LP: lumbar puncture/spinal tap

\section{Genetics}

$A D$ : autosomal dominant

AR: autosomal recessive.

p: short arm of a chromosome

q: long arm of a chromosome

DNA: deoxyribonucleic acid

RNA: ribonucleic acid

NB: newborn/neonate

\section{Organizations}

AAN: American Academy of Neurology

AES: American Epilepsy Society

AAP: American Academy of Pediatrics

CAIE: Centros de Atención Integral para la Epilepsia or Comprehensive Care Centers for Epilepsy

FDA: Food and Drug Administration

IBE: International Bureau for Epilepsy

ILAE: International League Against Epilepsy

INNN: Instituto Nacional de Neurología y Neurocirugía or National Institute for Neurology and Neurosurgery.

WHO: World Health Organization

PAHO: Pan American Health Organization

PPE: Programa Prioritario de Epilepsia or Priority Epilepsy Program

SAdE: Sociedad Andaluza de Epilepsia or Andalusian Epilepsy Society
GPC-PPE: Guía de Práctica Clínica del Programa Prioritario de Epilepsia or Clinical Guidelines of the Priority Epilepsy Program

\section{Additional neurological disorders}

CVD: cerebrovascular disease

TBI: traumatic brain injury

\section{Drug administration routes}

IM: intramuscular

IV: intravenous

PO: oral

S/C: subcutaneous

S/L: sublingual

\section{Syndromes and types of epilepsy}

BECTS: benign epilepsy with centrotemporal spikes (Rolandic Epilepsy)

IGE: idiopathic generalized epilepsy

PME: progressive myoclonus epilepsy

JME: juvenile myoclonus epilepsy

MTS: mesial temporal sclerosis

LGS: Lennox-Gastaut syndrome

DRE: difficult to treat seizures/drug-resistant epilepsy

\section{Miscellaneous}

AED: antiepileptic drugs/anti-seizure medications

BZD: benzodiazepines

\section{Antiepileptic drugs}

ACZ: acetazolamide

ACTH : adrenocorticotropic hormone

NE: barbexaclone

$\mathrm{NE}$ : beclamide

CBZ: carbamazepine

CLB: clobazam

CZP: clonazepam

CLP: clorazepate

DZP: diazepam

ESM: ethosuximide

FBM: felbamate

GBP: gabapentin

LTG: lamotrigine

LEV: levetiracetam

LZP: Iorazepam

MDL: midazolam 
MPH: methylphenidate

MPB: methylphenobarbital

MSM: mesuximide/methsuximide

NTZ: nitrazepam

OXC: oxcarbazepine

PAC: phenacemide

PTR: pheneturide

PB: phenobarbital

PSM: phensuximide

PHT: phenytoin

NE: fosphenytoin

PGB: pregabalin

PRM: primidone

PRO: progabide

STM: sultiame/sulthiame

TGB: tiagabine

TPM: topiramate

$\mathrm{NE}$ : trimethadione

VPA: valproic acid

VGB: vigabatrin

ZNS: zonisamide

NE: 4-amino-3-hydroxibutiric Acid

FLN: flunarizine

LSG: losigamone

RLT: ralitoline (Cl-946)

REM: remacemide

STP: stiripentol

HRK: harkoseride

LCM: lacosamide

RET: retigabine (D-23129)

BRV: brivaracetam

STM: seletracetam (ucb 44212)
CBT: carabersat (SB-204269)

TBT: tonabersat (SB-220453)

SFM: safinamide (PNU-151774E)

RUF: rufinamide (SGP33101)

STL: soretolide (D-2916)

TLP: talampanel (GYKI 53773)

HUP: huperzine $A$

ATM: atipamezole

VLR: valrocemide (TV1901)

IVR: isovaleramide

VPG: valproyl glycinamide

VLT: valnoctamide

VPD: valpromide

VCD: valrocemide

PID: propylisopropylacetamide

LiCBZ: licarbazepine

EsliCBZ: eslicarbazepine (BIA 2-093)

FI-FBM: fluorofelbamate

NA: ganaxolone

carisbamate (RWJ-333369)

perampanel

ELB-139

JZP-4

NS-1209

CGX-1007

SPD-421

ICA27243

T2000

XP-13512*

YKP3089

NE: not established

NA: not applicable 


\section{Centers of integral care for epilepsy "CAIE" in Mexico}
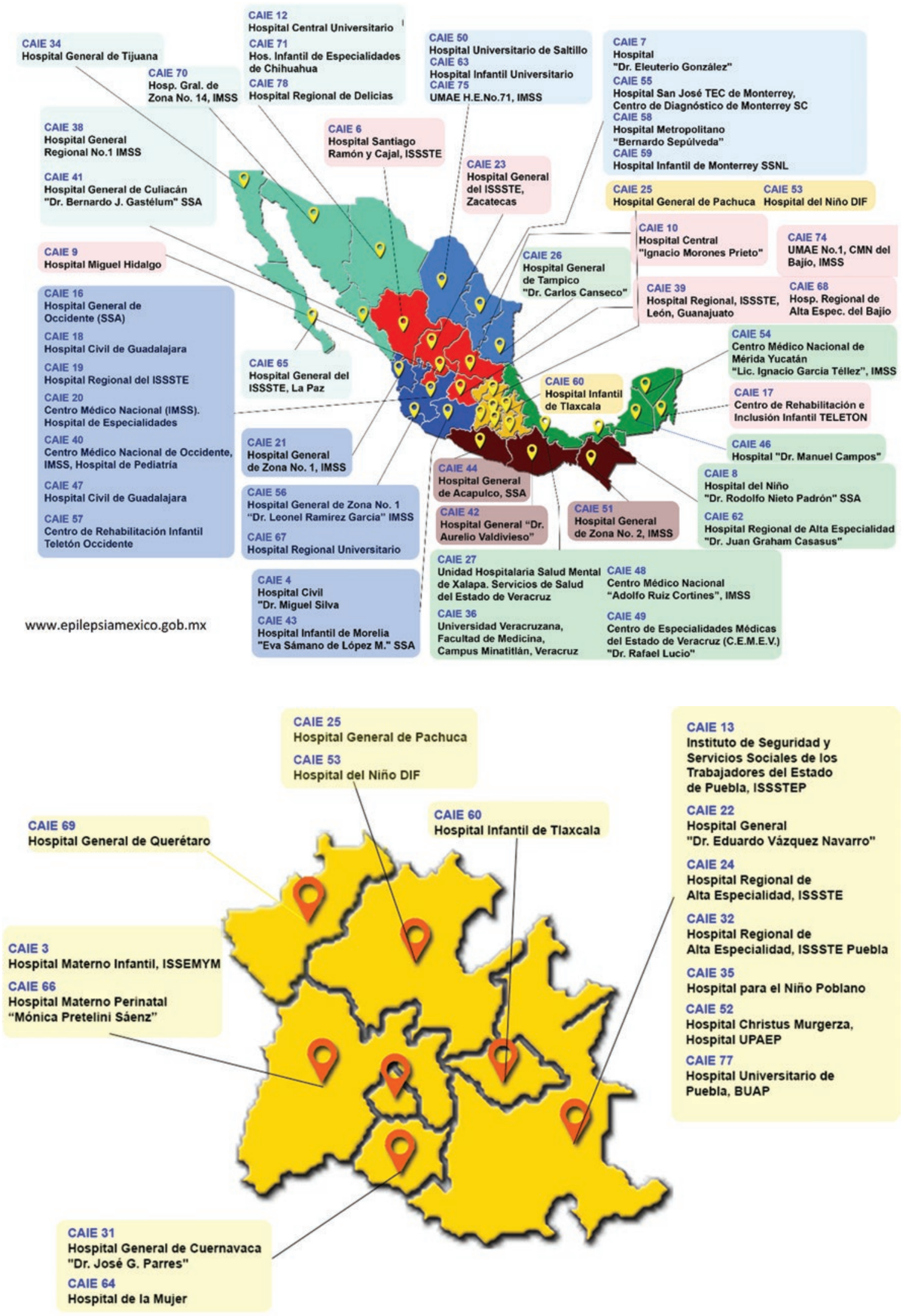

www.epilepsiamexico.gob.mx 
CAIE 1.- Instituto Nacional de Neurología y Neurocirugía.

CAIE 2.- Instituto Nacional de Pediatría.

CAIE 5.- Hospital Pediátrico de Legaria.

CAIE 11.- Instituto Nacional de Perinatología.

CAIE 14.- Hospital General Centro Médico La Raza. Hosp. Gral. "Dr. Gaudencio González Garza”, IMSS.

CAIE 15.- Centro Médico Nacional "20 de Noviembre".

CAIE 28.- Hospital Psiquiatrico Infantil "Dr. Juan N. Navarro".

CAIE 29.- Hospital Central de Alta Especialidad Sur PEMEX.

CAIE 30.- Hospital Infantil de México "Federico Gómez".

CAIE 33.- Centro Médico Nacional "20 de Noviembre", ISSSTE.

CAIE 37.- Hospital General de México "Dr. Eduardo Liceaga", Unidad de Pediatría.

CAIE 45.- Instituto Nacional de Ciencias Médicas y Nutrición "Salvador Zubirán".

CAIE 61.- Hospital General "Dr. Manuel Gea González".

CAIE 72.- Hospital Regional "Lic. Adolfo López Mateos", ISSSTE.

CAIE 73.- Unidad Médica de Alta Especialidad, Hospital de Pediatría "Dr. Silvestre Frenk Freund" CMN Siglo XXI, IMSS.

CAIE 76.- Hospital Central Norte, PEMEX.

www.epilepsiamexico.gob.mx

Thanks to Jetzabel Fragoso and Dr. Francisco López for their support in all the activities of the Priority Epilepsy Program. 